\title{
Management of chronic migraine with medication overuse by web-based behavioral program during the COVID-19 emergency: results at 12 months
}

\author{
Licia Grazzi ${ }^{1} \cdot$ Alessandra Telesca $^{1,2} \cdot$ Paul Rizzoli $^{3}$
}

Received: 26 July 2021 / Accepted: 15 October 2021 / Published online: 21 December 2021

(c) Fondazione Società Italiana di Neurologia 2021

\begin{abstract}
Background The study had been initiated because of restrictions put in place to control the spread of coronavirus in Milan in March 2020 that impacted clinical activities at our tertiary headache center in Milan (Foundation IRCSS Carlo Besta Neurological Institute). Treatment efforts were modified to make use of telephonic and internet communication to maintain care of our patients.

Methods Nineteen patients had undergone our withdrawal protocol for medication overuse headache and were scheduled for follow-up that included pharmacological prophylaxis combined with behavioral therapy and mindfulness, generally performed in small group face-to-face sessions. A behavioral program was organized for them by technology modality (smartphone) due to the pandemic restrictions.

Results The results concern 12-month follow-up. The clinical indexes showed migraine days per month $20.6( \pm 6)$ pre vs 11.2 ( \pm 3.1 at 12 months); medications intake per month $19.4( \pm 5.8)$ pre vs $9.0( \pm 4.6)$ at 12 months; MIDAS $67.7( \pm 52.6)$ pre vs $71.1( \pm 60)$ at 12 months; HIT-6 $66.2( \pm 5.3)$ pre vs $62.2( \pm 5.9)$.

Conclusion The present study confirmed literature data, supporting the behavioral approach combined to traditional therapies as a novel method to follow patients and guarantee their benefit, also when applied using technology by telemedicine or smartphone.
\end{abstract}

Keywords Chronic migraine $\cdot$ Medication overuse $\cdot$ Mindfulness $\cdot$ Telemedicine $\cdot$ COVID-19 emergency

This brief communication provides follow-up results of a clinical experience concerning patients with chronic migraine and medication overuse and the suspension of usual treatment protocols during the March 2020 COVID19 pandemic [1] in Italy and results at 12 months. We previously reported 6-month results [2].

The study had been initiated because of restrictions put in place to control the spread of coronavirus in Milan in March

Licia Grazzi

Licia.grazzi@istituto-besta.it

1 Dipartimento Neuroalgologia Centro Cefalee, Fondazione IRCCS Istituto Neurologico C.Besta, Milan, Italy

2 University of Milano-Bicocca, Milan, Italy

3 Department of Neurology, Brigham and Women's Faulkner Hospital, Brigham and Women's Hospital, John Graham Headache Center, Harvard Medical School, Boston, MA, USA
2020 that impacted clinical activities at our tertiary headache center in Milan (Foundation IRCSS Carlo Besta Neurological Institute) [1]. Because of these restrictions, treatment efforts were modified to make use of telephonic and internet communication to maintain care of our patients.

Nineteen patients who had undergone our withdrawal protocol for medication overuse headache were scheduled for follow-up.

Our usual follow-up management includes pharmacologic prophylaxis, started after withdrawal, combined with behavioral therapy, in particular mindfulness, generally performed in small group face-to-face sessions, to help patients to become more conscious about their pain and learn techniques for managing pain without medications [1]. Pharmacologic preventive therapies were selected from among the traditional oral options for migraine: topiramate, amitriptyline, beta blockers, according to the general characteristics of patients and their migraine history. The new 
anti-calcitonin gene-related peptide monoclonal antibodies were not employed as they were not available in our center at the time.

The behavioral program, originally performed in person in our center, was switched to live weekly internet presentations by a mindfulness expert, supplemented by a brief (10-12 min) recorded home exercise, downloaded to the patient's smartphone, and meant for daily use for mindfulness practice.

Four patients decided to continue with the pharmacological therapy only without the behavioral program. These patients were discharged to be followed in headache centers closer to their homes and their data were not available. One patient dropped out for unrelated medical issues, leaving 14 patients in the program ( 3 males/ 11 females; mean age $45.2 \pm 8.9$; onset of disease $21.2 \pm 10.9)$ who completed the 6-month and the 12-month follow-up (Table 1). Headache days and medications were recorded monthly. Because of the small sample size, a descriptive statistical analysis was performed on the obtained results.

At 6 months, migraine days were reduced: $20.6( \pm 6.04)$ versus $8.7( \pm 4.5)$. Medication intake was reduced from $19.4( \pm 5.8)$ versus $7.5( \pm 5.0)(p=0.0002$ and $p=0.000001$ respectively). Questionnaires demonstrated a decrease in disability: MIDAS score from $67.7( \pm 52.6)$ vs 35.1 $( \pm 36.5)$; HIT-6 from $66.2( \pm 5.3)$ vs $60.0( \pm 7.6)(p=0.03$ and $p=0.02$ respectively) (Table 2 ).

At 12 months, the clinical indexes showed migraine days per month $20.6( \pm 6)$ pre vs $11.2( \pm 3.1)$ at 12 months; medications intake per month $19.4( \pm 5.8)$ pre vs $9.0( \pm 4.6)$ at 12 months ( $p=0.0002$ and $p=0.00004$ respectively); MIDAS $67.7( \pm 52.6)$ pre vs $71.1( \pm 60)$ at 12 months; HIT-6 $66.2( \pm 5.3)$ pre vs $62.2( \pm 5.9)$ at 12 months ( $p$ ns and $p 0.04$ respectively) (Table 2 ).

Though adherence was not formally measured, most patients participated actively in the program and did not miss any sessions or video calls. Patients reported that they were comfortable with this program and felt they had been carefully followed using this treatment modality [2].

The results we obtained with this novel application of our behavioral treatment during this dramatic emergency were

Table 1 General characteristics of patients

\begin{tabular}{ll}
\hline Number of patients enrolled & 19 \\
\hline Patients completing the program & $14(3 \mathrm{M} / 11 \mathrm{~F})$ \\
Mean age & $45.2( \pm 8.9)$ \\
Onset of disease & $21.2( \pm 10.9)$ \\
Patients dropped & $5^{*}$
\end{tabular}

*One patient reported dropped out for unrelated medical issues; 4 patients preferred to follow only the pharmacological program (they preferred not to use devices) and were followed in other headache centers located closer to home
Table 2 Clinical results and questionnaire values pretreatment and at 6- and 12-month follow-up

\begin{tabular}{llll}
\hline Migraine days/month & $20.6( \pm 6.04)$ & $8.7( \pm 4.5)$ & $11.2( \pm 3.1) *$ \\
\hline $\begin{array}{l}\text { Medication intake / } \\
\text { month }\end{array}$ & $19.4( \pm 5.8)$ & $7.5( \pm 5.0)$ & $9.0( \pm 4.6) * *$ \\
MIDAS score & $67.7( \pm 52.6)$ & $35.1(36.5)$ & $71.1( \pm 60) * * *$ \\
HIT-6 & $66.2( \pm 5.3)$ & $60.0( \pm 7.6)$ & $62.2( \pm 5.9) * * * *$ \\
\hline
\end{tabular}

* $p 0.0002$ pre vs $6 \mathrm{~m} ; p 0.0002$ pre vs $12 \mathrm{~m}$

${ }^{* *} p 0.00001$ pre vs $6 \mathrm{~m} ; p 0.00004$ pre vs $12 \mathrm{~m}$

**** $p 0.03$ pre vs $6 \mathrm{~m} ; p$ ns pre vs $12 \mathrm{~m}$

**** $p 0.02$ pre vs $6 \mathrm{~m} ; p 0.04$ pre vs $12 \mathrm{~m}$

A satisfaction questionnaire (SQ): completed at the end of the program, demonstrated a high level of acceptance of this modality from the patients. A six-question evaluation test easy to complete concerning the following: the quality of the program; the expectations respect to the program; the response to the needs of this program; possible suggestions to other patients about this specific program; level of help that the program deserved to patients; level of satisfaction

encouraging, with a significant change in monthly migraine days and medications intake in this group of patients at 12 months.

The disability score reported by the MIDAS questionnaire and the impact reported by the HIT- 6 questionnaire demonstrated a decrease at 6 months and returned to baseline at 12 months. This finding is unexplained and its significance, given the small sample size, is unclear. One consideration could be related to the dramatic pandemic condition that patients faced during these months. It also may be that outlier patients with persistent high disability scores impacted the overall results in this small sample size. However, this finding deserves further investigation.

The suspension of the usual program related to pandemic restrictions led to both patient anxiety and a significant concern for failure of the withdrawal therapy. This necessitated the creation of this specific "emergency protocol," using technology for mindfulness sessions and video calls, to meet patients' needs and to continue the therapeutic process started with the withdrawal treatment. Prior studies [3-5] have demonstrated the efficacy of telemedicine/technology in various clinical applications, including migraine [5, 6]. The present study confirmed literature data [7], supporting the behavioral approach combined to traditional therapies as a novel method to follow patients and guarantee their benefit.

Some limitations of this project are as follows: due to the emergency, the program was organized in a very short time which prevented, among other things, the randomization of patients, addition of an active comparator, addition of a control group and adequate group size; another possible limitation could be a selection bias: the $30 \%$ drop rate could include patients who preferred not to participate to this type of management. 
Nevertheless, the 6-month follow-up data and, more important, the 12-month clinical results provide useful information about the effectiveness of this program. Though preliminary and in a restricted group of patients, the results are encouraging telemedicine and the use of technology by smartphone since they indicate both clinical effectiveness combined with high patients' adherence. The long period of follow-up contributes to confirm clinical results.

This approach could be considered a first step toward a new era of patient care that maintains efficacy while conserving time and resources for both patients and providers.

The study received Ethic Committee approval in March 2020 (a special session of Ethic Committee was performed after the STAY-AT-HOME announcement from the Italian Governor); also, the study was registered in ClinicalTrials. gov ID: NCT04389372. Patients signed a written consent to participate to the study according to this modality.

\section{Declarations}

Ethical approval The program was approved by the Ethic Committee of the Besta institute on May 2020; also the program was submitted and accepted by TRIALGOV (see previous publication on Headache, January, 2021).

Informed consent Patients were informed of the program and they put their signature on informed consent form.

\section{Conflict of interest None}

\section{References}

1. Grazzi L, Rizzoli P (2020) The adaptation of management of chronic migraine patients with medication overuse to the suspension of treatment protocols during the COVID-19 pandemic: lessons from a tertiary headache center in Milan, Italy. Headache 60:1463-1464

2. Grazzi L, Rizzoli P (2021) Adaptation of management of chronic migraine patients with medication overuse to the suspension of treatment protocols during the COVID-19 pandemic: lessons from a tertiary headache center in Milan-6 months results. Headache 61:961-962

3. Eccleston C, Blyth F, Dear B et al (2020) Managing patients with chronic pain during the COVID-19 outbreak: considerations for the rapid introduction of remotely supported (eHealth) pain management services. Pain 161(5):889-893

4. Qutby W, Patniyot I, Gelfand A (2018) Telemedicine in a pediatric clinic. A prospective survey. Neurology 90(19):1-7

5. Linardon J (2020) Can acceptance, mindfulness and self-compassion be learned by smartphone and apps? A systematic metaanalytic review of randomized controlled trials. Behav Ther 51:646-658

6. Friedman D, Rajan B, Seidmann A (2019) A randomized trial of telemedicine for migraine management. Cephalalgia 39(12):1577-1585

7. Grazzi L, Raggi A, D'Amico D, Sansone E, Leonardi M, Andrasik F, Gucciardi A, Guido D, D'Andrea G (2018) A prospective pilot study of the effect on catecholamines of mindfulness training vs pharmacological prophylaxis in patients with chronic migraine and medication overuse headache. Cephalalgia Sep 13:333102418801584. https://doi.org/10.1177/0333102418 801584

Publisher's note Springer Nature remains neutral with regard to jurisdictional claims in published maps and institutional affiliations. 